\title{
Strain Gauge Sensors Comprised Of Carbon Nanotube Yarn: Concept and Modeling
}

\author{
Jandro L. Abot ${ }^{1}$, Emílio C. N. Silva ${ }^{2}$, César Y. Kiyono ${ }^{2}$, Gilles P. Thomas ${ }^{2}$ \\ ${ }^{1}$ Department of Mechanical Engineering, The Catholic University of America, Washington DC, 20064, \\ USA, abot@cua.edu \\ ${ }^{2}$ Department of Mechatronics and Mechanical Systems Engineering, School of Engineering, University \\ of São Paulo, São Paulo,SP - 05508-900,Brazil, ecnsilva@usp.br, ckiyono@gmail.com, \\ gillespierre.thomas@gmail.com
}

\begin{abstract}
Carbon nanotube yarns are micron-size fibers that contain thousands of intertwined carbon nanotubes in their cross sections and exhibit piezoresistance characteristics that can be tapped for sensing purposes. Sensor yarns can be integrated in polymeric and composite materials to measure strain through resistance measurements without adding weight or altering the integrity of the host material. This paper includes the details of novel strain gauge sensor configurations made of carbon nanotube yarn and the modeling of their piezoresistive response using parametric optimization schemes that maximize the sensor' sensitivity to mechanical loading. The effect of several sensor configuration parameters are discussed including the angle and separation of the carbon nanotube yarns within the sensor, and also the mechanical properties of the CNT yarns.
\end{abstract}

Keywords: Strain Gauge, Carbon Nanotube Yarn, Piezoresistive Response, Modeling, Parametric Optimization

\section{Introduction}

Most existing strain sensors may alter the material's microstructure and some may compromise the integrity or reliability of the structure by requiring too many sensors or complex equipment to acquire the strain gauge data. The ideal strain gauge should be small, low in cost, easily integrated, and highly sensitive to strain but insensitive to ambient or process temperature variations. Carbon nanotube (CNT) yarns are micron-size fibers that contain thousands of intertwined carbon nanotubes in their cross sections and exhibit piezoresistance characteristics that can be tapped for sensing purposes $[1,2]$. The use of strain gauge sensors composed of CNT yarn may offer a feasible and practical measurement of strain in polymeric and composite materials [3-5]. Piezoresistive strain gauge sensors are typically made of a piezoresistive membrane layer attached to a flexible substrate layer. The flexible structure works as a compliant mechanism that converts an input force into local strain and stress in the piezoresistive layer so that changes in resistivity can be monitored as changes in output voltage, which can be correlated to the strain using the piezoresistive effect. The piezoresistive layer is electrically connected to a Wheatstone bridge, which increases the sensor sensitivity and compensates for temperature effects [6]. A set of systematic design methodologies are available for designing piezoresistive sensors. For example, a parametric optimization methodology can be conducted to design piezoresistive strain gauge sensors. Typically, the design goal is to obtain the location and configuration of a material that maximizes the sensor sensitivity to external loading. This paper presents novel concepts of strain gauges comprising CNT yarn and includes the details of the modeling of their piezoresistive response. 


\section{Sensor concepts and configurations}

An image of the 1-thread CNT yarn is presented in Figure 1a. This CNT yarn has a diameter of about 30 microns and the mechanical and electrical properties are similar to those provided elsewhere [5]. A schematic of the strain gauge configuration including the piezoresistive layer containing the CNT yarns, the substrate composed of a polymeric material, and the electrodes is shown in Figure 1b. The "building block" or basic initial configuration assumed for the piezoresistive layer is an arrangement of parallel CNT yarns as shown in the inset of Figure 1b. A parametric optimization will be conducted on this building block to explore the use of various sensor shapes including rectangular and cross-like configurations (Figure 1c). The sensor configurations that provide the best sensitivity are obtained from the parametric optimization process and the corresponding modeling formulation and results are presented next.

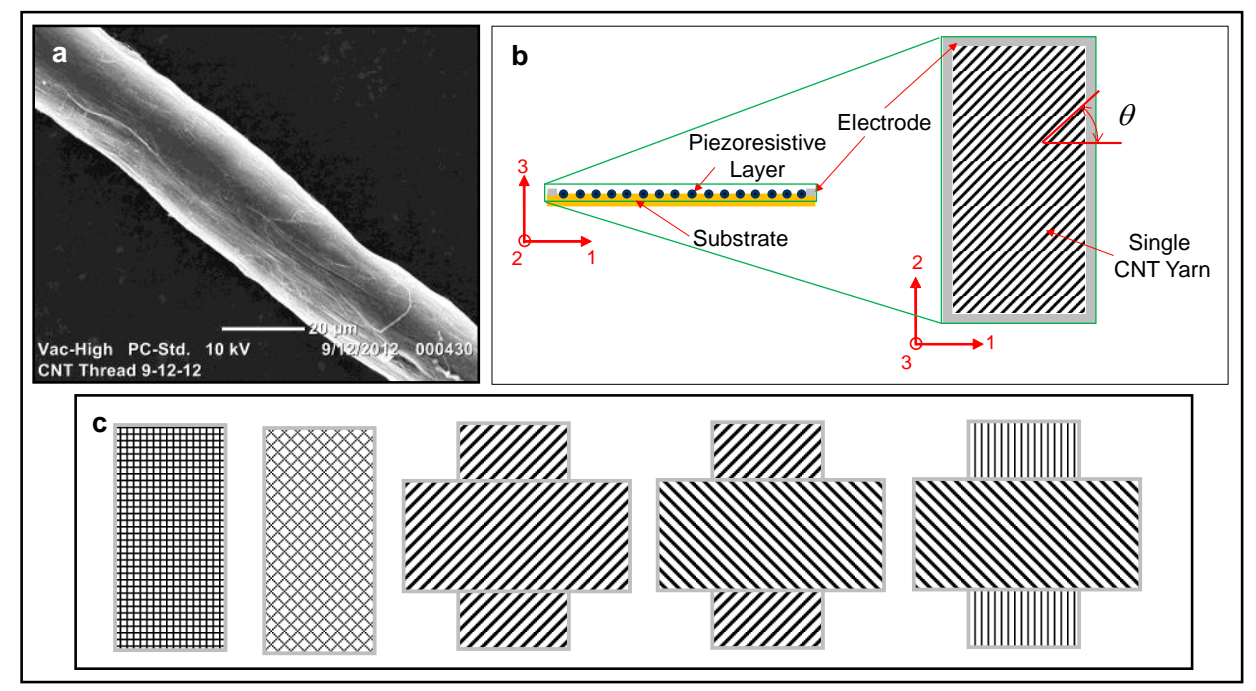

Figure 1: (a) Scanning Electron Microscope image of a 1-thread CNT yarn. (b) Schematic view of the configuration of the strain gauge sensor. Inset: building block showing the arrangement of the CNT yarns. (c) Top view schematic of various sensor configurations used in the modeling effort.

\section{Modeling}

\subsection{Formulation and numerical implementation}

A parametric optimization and analysis was implemented in Matlab ${ }^{\mathrm{TM}}$ and ANSYS ${ }^{\mathrm{TM}}$ to design four-terminal strain gauge sensors containing a piezoresistive layer of CNT yarns. The analysis was performed using the Finite Element Method (FEM) and the resulting FEM linear system of equations was obtained using the method of weighted residuals [7]. The sensitivity, given by the output voltage of the strain gauge sensor, was studied by varying a series of geometrical and material parameters. This mechanical-electrical analysis requires knowing three material properties including the elastic modulus, the electrical resistivity and the piezoresistive response of the CNT yarn [5]. The former two are constant and the latter is nonlinear. The differential equations that govern the electrical and mechanical response of the sensor are presented elsewhere including the assumptions for the FEM formulation [8].

The model considered a single layer where the piezoresistive layer composed of the CNT yarns is immersed in the polymer substrate. In order to create a uniform state of strain in the sensor, a formulation is implemented where the strain sensor is placed in a medium that is subjected to a far field stress in one side and a fixed boundary condition on the other side, as seen in Figure 2 [9,10]. This allows the sensor to experience the same longitudinal strain, $\varepsilon_{x x}$, and transverse strain, $\varepsilon_{y y}$, fields as the host material. An analytical model was also developed based on a previous analysis [9] and slightly modified to account for the specific configuration of the strain gauge in this study. The results of this analytical model confirm the numerical results presented in the next section [8]. 


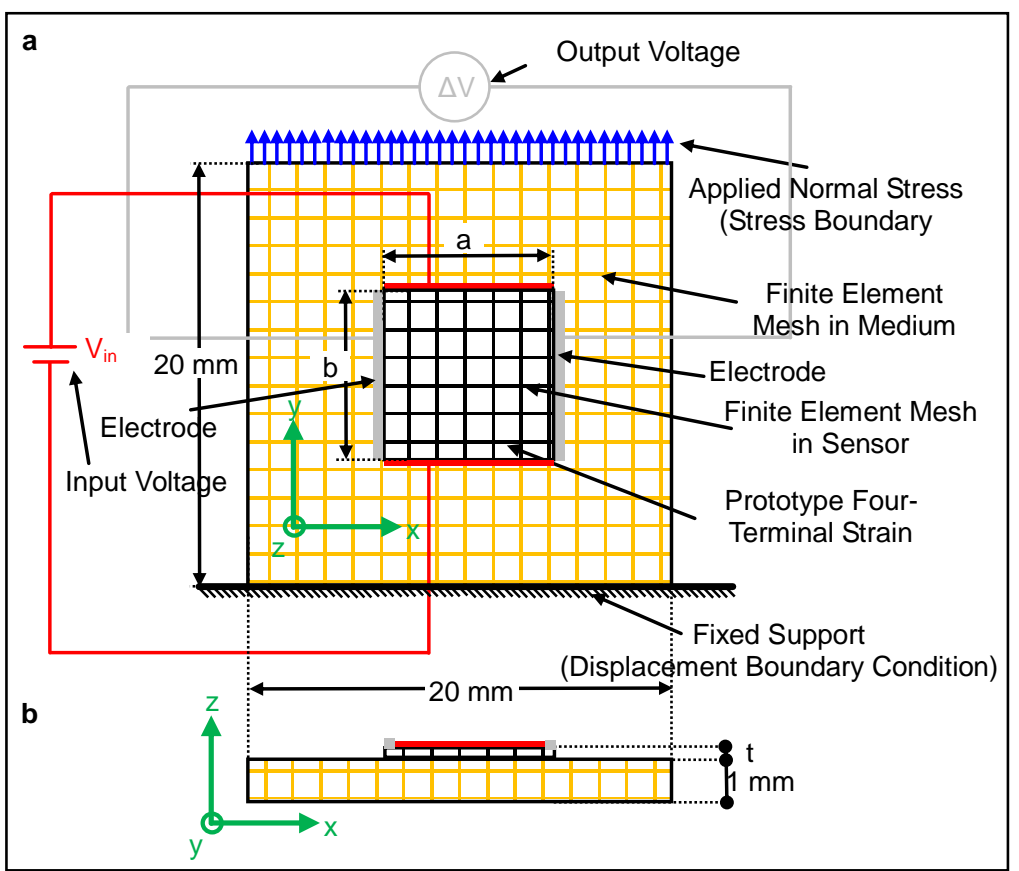

Figure 2: Top view and lateral schematic views of the sensor subjected to a far field stress including finite element mesh, boundary conditions, and input and output voltage details.

\subsection{Modeling results}

The modeling results of the parametric optimization and analysis of the sensor are presented and discussed next. The sensitivity of the sensor is calculated as the difference in the output voltage between the case of applied mechanical stress and the case of no stress and divided by the latter. The four-terminal sensor configuration allows it to have a Wheatstone bridge inside the strain gauge, and thus enable the leveraging of the voltage reading. The shape and dimensions of the sensor are very critical in the case of this strain gauge composed of CNT yarns. Regular metallic foil strain gauges have a configuration such that the each foil has a high aspect ratio to minimize the effect of the transverse strain. Initially, a square sensor with dimensions of $10 \mathrm{~mm}$ by $10 \mathrm{~mm}$ was considered and subjected to mechanical loading (applied stress, $\sigma_{\mathrm{yy}}$, of $100 \mathrm{MPa}$ ) and electrical loading ( $1 \mathrm{~V}$ between the electrodes) and oriented at $0^{\circ}$ with its sides parallel to the sides of the medium as shown in Figure 2. The longitudinal normal strain field, $\varepsilon_{y y}$, in the sensor was observed to be uniform everywhere and the transverse normal strain in the sensor was almost uniform inside the sensor except for a few locations at the corners. The shear strain field exhibited a small gradient inside the sensor but its magnitude remained one order of magnitude below that of the longitudinal normal strain.

The effect of various parameters on the sensitivity of the sensor was studied including the orientation and spacing of the CNT yarns within the sensor and the value of the Poisson's ratio of the CNT yarn. The effect of other parameters such as the configuration of the CNT yarns including an orthogonal mesh, the direction of the applied mechanical stress, and the dimensions and shape of the sensor are presented elsewhere [8]. For example, the effect of the CNT yarn orientation within a square strain gauge at various stress levels is shown in Figure 3a. It is observed that the relative voltage change varies significantly with the orientation of the CNT yarns. It increases sharply between $0^{\circ}$ and $45^{\circ}$, decreases relatively gradually between $45^{\circ}$ and $75^{\circ}$, because many CNT yarns touch both the upper and lateral electrodes, and then decreases more sharply between $75^{\circ}$ and $90^{\circ}$. The sensitivity as a function of the CNT yarn orientation is presented in Figure $3 \mathrm{~b}$. The curves, parametric on the stress level, exhibit a quasi-sinusoidal response with relative peaks at about $7^{\circ}, 45^{\circ}$ and $70^{\circ}$. The effect of the stress level is almost linear and only deviates in the case of the higher stress level curve, in the range between $45^{\circ}$ and $85^{\circ}$, increasing the sensitivity even further. The sensitivity reaches a maximum value at an angle of $70^{\circ}$ regardless of the stress level reaching a value of about $2 \times 10^{-3}$ when the stress level is $1 \mathrm{GPa}$. The effect of the CNT yarn spacing within a square sensor parametrically on various stress levels is shown in Figure $4 \mathrm{a}$. The maximum sensitivity is obtained when the separation between the CNT yarns is minimal. The sensitivity decreases asymptotically reaching an almost constant value at about 10 diameters. The effect of the Poisson's ratio of the CNT yarn on the square sensor of specific dimensions is shown in Figure 4b. Since the actual Poisson's ratio of the CNT yarn in this study was unknown, the value was varied between 0 and 8 . It is observed that this parameter plays a significant role in the sensitivity, which increases almost linearly for high stress levels such as $1 \mathrm{GPa}$. The sensitivity reaches a value of about $2.7 \times 10^{-3}$ for a Poisson's ratio of 8 and a stress level of $1 \mathrm{GPa}$. 

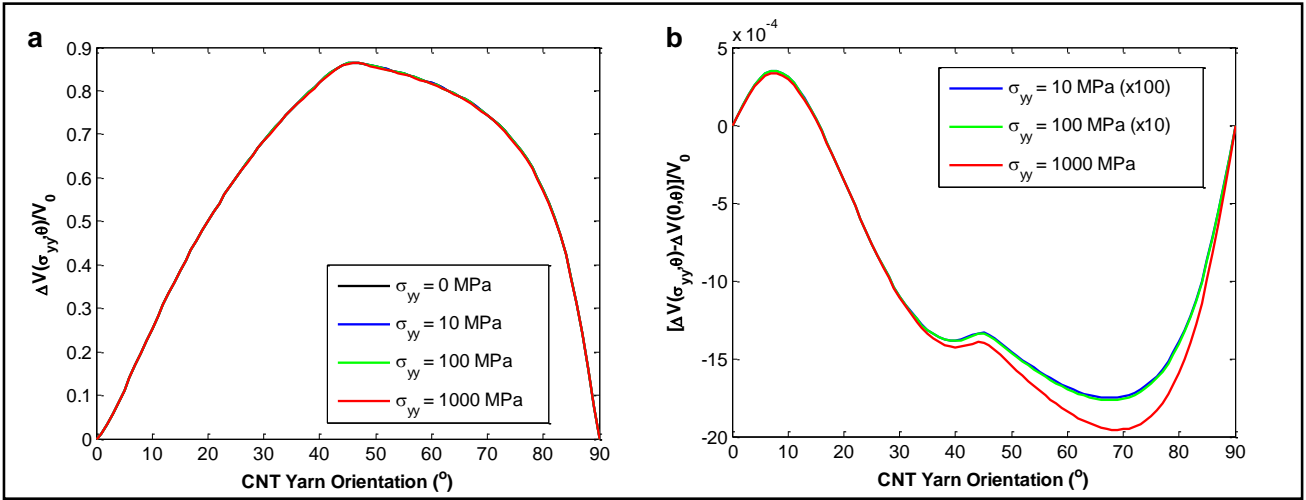

Figure 3: (a) Effect of CNT yarn orientation on the relative voltage change of the sensor (Square sensor of side a=10 mm; Sensor orientation $=0^{\circ}$; Vin $=1 \mathrm{~V} ; \sigma_{\mathrm{yy}}=$ variable; CNT yarn spacing $=1 \Phi ; v=6$ ); (b) Effect of CNT yarn orientation on the sensitivity of the sensor (Square sensor of side $\mathrm{a}=1 \mathrm{~cm}$; Sensor orientation $=0^{\circ}$; Vin $=1 \mathrm{~V} ; \sigma_{\mathrm{yy}}=$ variable; CNT yarn spacing $=1 \Phi ; v=6$ ).
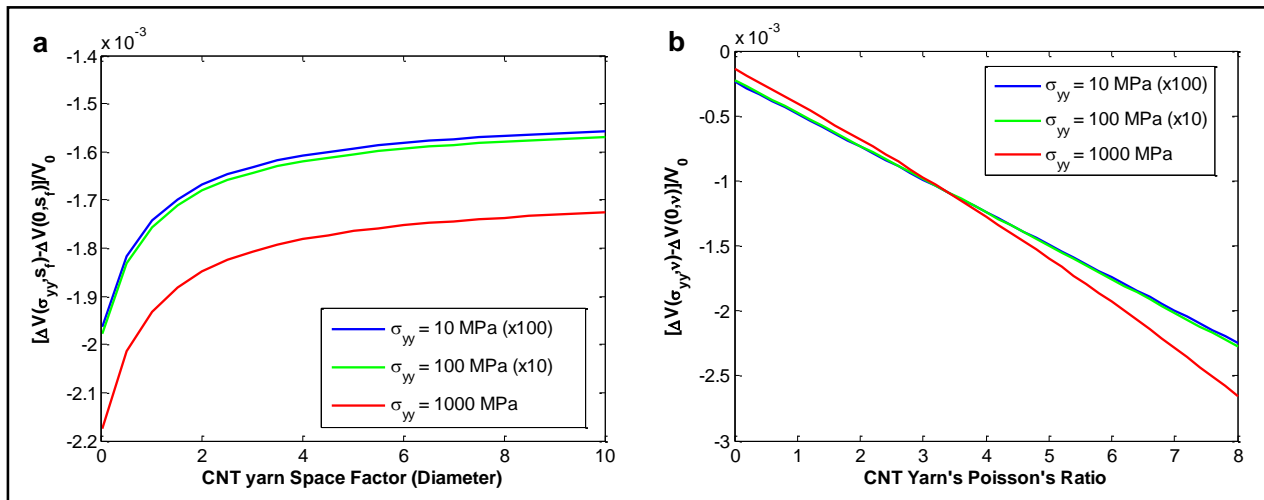

Figure 4: (a) Effect of CNT yarn spacing on the sensitivity of the sensor (Square sensor of side a=10 mm; Sensor orientation= $0^{\circ}$; Vin=1 V; $\sigma_{y y}=$ variable; CNT yarn orientation $=65^{\circ}$; CNT yarn spacing=variable; $v=6$ ); (b) Effect of Poisson's ratio on the sensitivity of the sensor (Square sensor of side $\mathrm{a}=10 \mathrm{~mm}$; Sensor orientation $=0^{\circ}$; Vin $=1 \mathrm{~V} ; \sigma_{\mathrm{yy}}=$ variable; $\mathrm{CNT}$ yarn orientation $=65^{\circ} ; \mathrm{CNT}$ yarn orientation $=0^{\circ}$; $\mathrm{CNT}$ yarn spacing $=1 \Phi ; v=$ variable).

From all the previous results, it can be concluded that the highest sensitivity in the case of a square sensor would be achieved with CNT yarns oriented at about $70^{\circ}$ and spaced as close to each other as possible. Modeling results are also available for rectangular and cross shape sensors [8]. Also, the sensor is sensitive to all stress components although the stress in the longitudinal direction, $\sigma_{\mathrm{yy}}$ plays the biggest role [8]. Crucially, the modeling results indicate that the sensors consisting of CNT yarn are sensitive enough to measure strain and that their most sensitive configurations exhibit even higher gauge factors than those of metallic foil strain gauges.

\section{Acknowledgements}

Abot acknowledges the financial support of this project by a 2014-2015 US Scholar Fulbright-Brazil Scientific Mobility Program grant.

\section{References}

1. K.L. Jiang, Q.Q. Li and S.S. Fan. Nanotechnology: spinning continuous carbon nanotube yarns. Nature, 419:801, 2002.

2. W. Lu, M. Zu,, J.-H. Byun, B.-S. Kim and T.-W. Chou. State of the art of carbon nanotube fibers: opportunities and challenges Advanced Materials, 24(14):1805-1833, 2012.

3. J.L. Abot, M.J. Schulz, Y. Song, S. Medikonda and N. Rooy. Novel distributed strain sensing in polymeric materials. Smart Materials and Structures, 19(8):085007, 2010.

4. A. Hehr, M. Schulz, V. Shanov and Y. Song. Embedded carbon nanotube thread piezoresistive strain sensor performance. Sensor Review, 34(2):209-219, 2014.

5. J.L. Abot, T. Alosh and K. Belay. Strain dependence of electrical resistance in carbon nanotube yarns. Carbon, 70:95-102, 2014.

6. J.W. Dally and W.F. Riley. Experimental Stress Analysis. McGraw-Hill, 1991.

7. J.N. Reddy. Theory and Analysis of Elastic Plates and Shells. CRC Press, 2006.

8. J.L. Abot, E.C.N. Silva, C.Y. Kiyono and G.P. Thomas. Strain gauge sensors comprised of carbon nanotube yarn: Parametric optimization modeling of their piezoresistive response. Smart Materials and Structures, (submitted).

9. L.A.M. Mello, C.Y. Kiyono, P.H. Nakasone and E.C.N. Silva. Design of quasi-static piezoelectric plate based transducers by using topology optimization. Smart Materials and Structures, 23(2):025035, 2014.

10. M.-H. Bao, W.-J. Qi and Y. Wang. Geometric design rules of four-terminal gauge for pressure sensors. Sensors and Actuators, 18:149-156, 1989. 